Ann. Zootech., I965, 14 (4), 39I-399.

\title{
L'ACCROISSEMENT EN EPAISSEUR DU TISSU GRAS DORSAL CHEZ LE VERRAT EN CROISSANCE
}

\author{
L. OLLIVIER \\ avec la collaboration technique de J. Saliou, I. Canope, D. Bralit et M. Renault \\ Station centrale de Génétique animale, \\ Centre national de Recherches zootechniques, Jouy-en-Josas (Seine-et-Oise)
}

\section{SOMMAIRE}

Un échantillon de 29 verrats de race Large White, placé dans les conditions d'une alimentation individuelle semi ad libitum, a été soumis à des mesures périodiques d'épaisseur du gras dorsal entre les poids de 40 et $100 \mathrm{~kg}$, à l'aide d'un appareil émetteur-récepteur d'ultrasons. La relation entre l'épaisseur du gras dorsal et le poids vif s'écarte significativement de la linéarité mais l'hypothèse d'une relation linéaire entre l'épaisseur du gras et le logarithme du poids vif est compatible avec les données. Il est possible, connaissant l'accroissement d'épaisseur du gras observé sur un verrat entre 40 et $80 \mathrm{~kg}$, de prédire l'épaisseur qu'il atteindra à ıoo $\mathrm{kg}$. La même relation peut également s'appliquer pour ramener à un poids donné des épaisseurs de gras mesurées à des poids variables.

\section{IN'TRODUCTION}

La proportion de tissu gras dans la carcasse du porc passe de $5 \mathrm{p}$. Ioo à environ 40 p. Ioo entre la naissance et le poids de Ioo $\mathrm{kg}$, d'après MC MEEKAN (r940). Cette évolution se traduit par un accroissement important de l'épaisseur du tissu gras sous-cutané, qu'il est possible de suivre sur l'animal vivant grâce à l'une des méthodes de mesure mises au point depuis ${ }_{9} 95^{2}:$ la réglette métallique de HAZEI, et $\mathrm{K}_{1, \mathrm{INE}}$ (I952), le leanmeter d'ANDREws et WhALEY (I954), la radiographie de Dumont et FÉvRIER (I957), l'appareil émetteur-récepteur d'ultrasons de DumonT (I957), Ces quatre moyens ont été utilisés pour étudier l'accroissement du gras dorsal sur le porc vivant respectivement par HETZER et coll. (I956), NOFFSINGER et coll. (r959). F́́vrier (r958), Urban et Hazei, (I960) ainsi que RíT'ter et coll. (I964). L'utili- 
sation des ultrasons présente l'avantage d'entraîner pour l'animal une perturbation minime et de permettre ainsi de nombreuses mesures sur le même individu. C'est la technique qui a été retenue pour cette étude dont le but est de suivre l'évolution de l'épaisseur du tissu gras dorsal chez le verrat en croissance.

\section{MATTÉRIEL, ANIMAL - MÉTHODES DE MESURE.}

Trente verrats en provenance d'un élevage inscrit au Her̂d-Bóok Làg ǵ White (') constituaient notre matériel expérimental. Ces trente verrats, issus de dix pères différents, ont été placés en cases individuelles à la station de mise à l'épreuve des verrats sur leur descendance, au C. N. R. Z.. Les animaux pesaient de 27 à $38 \mathrm{~kg}$ à leur arrivée à la station et de 97 à $105 \mathrm{~kg}$ en fin de contrôle. Un verrat cependant dut être éliminé à $89 \mathrm{~kg}$ et, par suite, exclu de cette étude. Sur les 29 verrats restants, 24 .ont été abattus et 5 gardés pour la reproduction.

L'alimentation, identique à celle distribuée aux animaux du contrôle de descendance $\left({ }^{2}\right)$, était distribuée selon l'appétit de l'animal, à raison de trois repas par jour.(alimentation semi ad libitum).

Tous les I4 jours, les animaux étaient pesés individuellement à jeun et sondés aux ultrasons. L'appareil utilisé, de marque 'Smiths, fabriqué en Grande-Bretagne, est calibré par les soins du constructeur et gradué directement en demi-centimètres. Les lectures étaient estimées au milljmètre près. Sur chaque animal, six emplacements étaient repérés avant le premier contrôle, permettant d'effectuer :

- 2 mesures à l'arrière de l'épaule, au niveau du "passage des sangles";

- 2 mesures au niveau de la dernière côte;

- 2 mesures au niveau du rein, à un emplacement déterminé par une verticale équidistante du grasset et de l'arrière des fesses.

Ces mesures étaient faites de part et d'autre de la ligne médiane, à environ $2 \mathrm{~cm}$ de cette ligne au début des contrôles. Ces emplacements correspondent approximativement, sur un porc de roo kg, à ceux proposés par HAZEL et KLINE (I952).

La découpe des carcasses était la découpe parisienne normalisée qui est utilisée dans les épreuves de descendance.

Les variablẹs retenues pour cette étude sont les suivantes :

io Sur l'animal vivant (29 verrats) :

- le poids vif;

- l'âge ;

- là moyennè deś six mesures d'épaisseur de gras.

Cette dernière variable a été ramenée, pour chaque individu, aux poids de 40,50,60,70,80, 90 et $100 \mathrm{~kg}$ par interpolation linéaire (et, éventuellement, extrapolation à $100 \mathrm{~kg}$ ). La moyenne des six épaisseurs de gras ainsi que le poids vif ont aussi été ramenés individuellement à des âges fixes, II 3,127 , I4I, I 55 et I69 jours, selon le même procédé.

$2^{0}$ Sur la carcasse (24 verrats abattus) :

- le pourcentage de morceaux nobles (jambon et longe) dans la demi-carcasse découpée;

- le pourcentage de morceaux gras (bardière et panne) dans la demi-carcasse découpée ;

- la moyenne de 2 épaisseurs de lard, mesurées l'une au niveau le la dernière vertèbre dorsale, l'autre au niveau de la dernière vertèbre sacrée.

(1) Élevage des Fromageries Bel.

(2) Composition de l'aliment.

Jusqu'au poids de $60 \mathrm{~kg}$

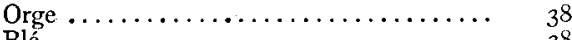

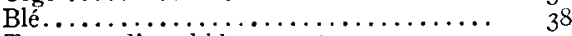

Tourteau d'arachide $\ldots \ldots \ldots \ldots \ldots \ldots \ldots$.

Tourteau de soja ................. 4

Son de blé..................... 5

Du poids de $60 \mathrm{~kg}$ au poids de $100 \mathrm{~kg}$

Orge $\ldots \ldots \ldots \ldots \ldots \ldots \ldots \ldots \ldots \ldots$ 4I

Blé....................... 42

Tourteau d'arachide............... 3

Tourteau de soja...................

Farine de luzerne..$\ldots \ldots \ldots \ldots \ldots \ldots \ldots$
Farine de poisson $\ldots \ldots \ldots \ldots \ldots \ldots \ldots \ldots$,

Farine de viande $\ldots \ldots \ldots \ldots \ldots \ldots \ldots \ldots \ldots$ 2

Levure de distillerie $\ldots \ldots \ldots \ldots \ldots \ldots \ldots .4$

Mćlange minéral ................. 3



Farine de viande $\ldots \ldots \ldots \ldots \ldots \ldots \ldots \ldots, \mathbf{z}$

Levure..$\ldots \ldots \ldots \ldots \ldots \ldots \ldots \ldots \ldots$ r $_{2}$

Mélange minéral ...................

Mélange vitaminique .............. - 


\section{RÉSULTATS}

Les résultats moyens des mesures sur le vivant sont rassemblés dans les tableaux I et 2 . I,es résultats moyens des mesures de carcasse, sur 24 verrats abattus, sont les suivants :

Pourcentage de morceaux nobles................ 54,3

Pourcentage de morceaux gras .................. I7,3

Épaisseur moyenne du lard au rein et au dos (mm) ..... 27,4

\section{TABLEAU I}

Évolution en fonction du poids de l'épaisseur du gras dorsal mesurée aux ultras sons (effectif de 29 verrats)

\begin{tabular}{c|c|c|c|c|c|c|c|c}
\hline \hline Poids $(\mathrm{kg})$ & 40 & 50 & 60 & 70 & 80 & 90 & 100 \\
\hline $\begin{array}{c}\text { Moyenne des six } \\
\text { mesures }(\mathrm{mm})\end{array}$ & $\begin{array}{c}14,41 \\
\pm 0,30\end{array}$ & $\begin{array}{c}18,28 \\
\pm 0,35\end{array}$ & $\begin{array}{c}20,98 \\
\pm 0,37\end{array}$ & $\begin{array}{c}24,07 \\
\pm 0,42\end{array}$ & $\begin{array}{c}26,55 \\
\pm 0,45\end{array}$ & $\begin{array}{c}28,67 \\
\pm 0,52\end{array}$ & $\begin{array}{c}30,61 \\
\pm 0,54\end{array}$ \\
\hline
\end{tabular}

\section{TABLEAU 2}

Évolution en fonction de l'âge et du poids vif de l'épaisseur du gras dorsal mesurée aux ultra sons (effectif de 29 verrats)

\begin{tabular}{|c|c|c|c|c|c|}
\hline Age (jours) & 113 & 127 & 141 & 155 & 169 \\
\hline Poids (kg) & $\begin{array}{r}40,86 \\
\pm \quad 0,59\end{array}$ & $\begin{array}{l}49,59 \\
\pm 0,73\end{array}$ & $\begin{array}{c}59,90 \\
\pm 0,73\end{array}$ & $\begin{array}{l}72: 49 \\
\div \quad 0,91\end{array}$ & $\begin{array}{r}8 \%, 90 \\
\pm \quad 1,04\end{array}$ \\
\hline $\begin{array}{c}\text { Moyenne de six mesures } \\
\text { de gras (mm) }\end{array}$ & $\begin{array}{c}14,87 \\
\pm 0,35\end{array}$ & $\begin{aligned} & 17,98 \\
- & 0,42\end{aligned}$ & $\begin{array}{c}21,12 \\
\pm 0,40\end{array}$ & $\begin{array}{c}24,74 \\
\pm 0,49\end{array}$ & $\begin{array}{c}27,63 \\
\pm 0,51\end{array}$ \\
\hline
\end{tabular}

I. Valeur des mesures effectuées sur l'animal vivant au poids d'abattage.

L'exactitude des mesures ultrasonores n'a pas été vérifiée directement. Seules ont été estimées les corrélations entre les résultats de la découpe d'une part et les mesures d'épaisseur du tissu gras effectuées soit sur le vivant, soit sur la carcasse d'autre part (tabl. 3). Il apparaît que les mesures effectuées sur le vivant sont en corrélation plus étroite avec les résultats de la découpe que les mesures sur la carcasse elle-même. 


\section{Relation entre poids, âge et épaisseur du gras dorsal.}

L'ajustement par les polynômes orthogonaux a donné les résultats du tableau 4 . Il est donc possible d'admettre une variation linéaire de l'épaisseur du gras en fonction de l'âge (entre II 3 et I69 jours) mais cette hypothèse est à écarter en ce qui

\section{TABLEAU 3}

Corrélations entre les résultats de la découpe et les mesures d'épaisseur du tissus gras (effectif de 24 verrats abattus).

\begin{tabular}{|c|c|c|}
\hline $\begin{array}{l}\text { Résultats } \\
\text { Mesures } \\
\text { d'épaisseur } \\
\text { du gras dorsal découpe }\end{array}$ & $\begin{array}{l}\text { Pourcentage de } \\
\text { jambon et longe } \\
\text { dans la carcasse }\end{array}$ & $\begin{array}{l}\text { l'ourcentage de } \\
\text { bardière et pann } \\
\text { dans la carcasse }\end{array}$ \\
\hline $\begin{array}{l}\text { Moyenne de } 6 \text { mesures sur le vivant } \\
\text { (au poids de } 100 \mathrm{~kg}) \ldots \ldots \ldots \ldots \ldots\end{array}$ & $\rightarrow 0,62$ & 0,82 \\
\hline $\begin{array}{l}\text { Moyenne de } 2 \text { mesures (au rein et } \\
\text { dos) sur la carcasse........... }\end{array}$ & $-0,15$ & 0,15 \\
\hline $\begin{array}{l}\text { Valeur du coeffirient de corrélat } \\
\text { l'hypothèse } p:-0\end{array}$ & aus & $\begin{array}{l}\text { de } 5 \%: 0,40 \\
\text { de } 1 \%: 0,52\end{array}$ \\
\hline
\end{tabular}

TABLEAU 4

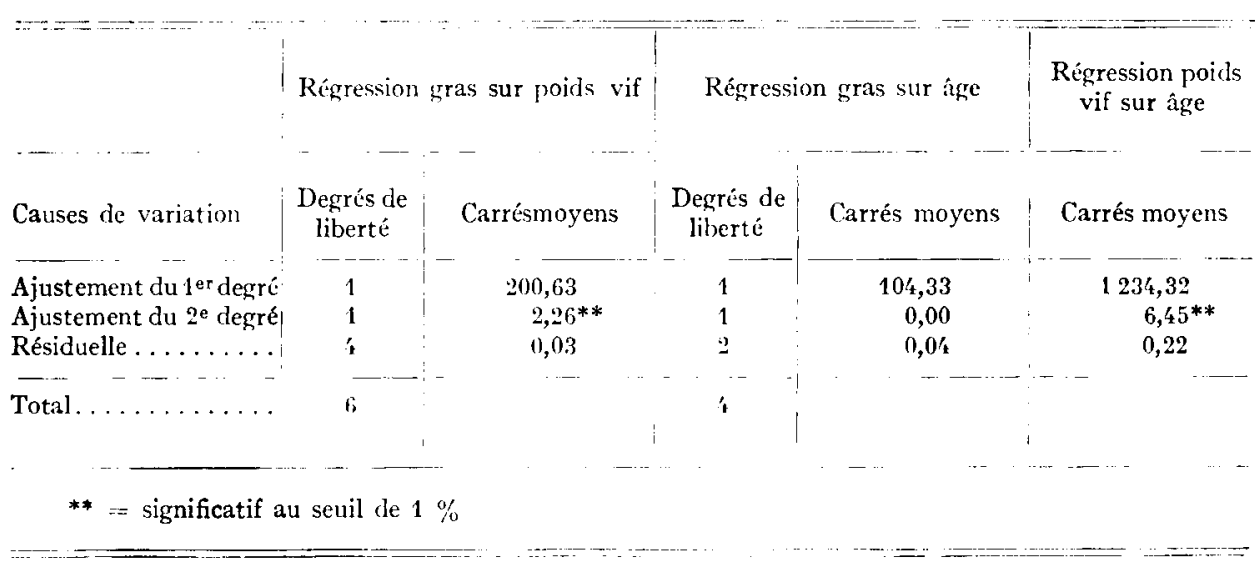

concerne d'une part la relation entre le poids et l'âge, d'autre part la relation entre l'épaisseur du gras et le poids. Le remplacement du poids par son logarithme, que suggère le caractère exponentiel de la croissance, permet d'ajuster 3 régressions 
linéaires entre ces variables (graph. I, 2 et 3). La somme des carrés résiduelle de la régression de l'épaisseur du gras sur le logarithme du poids est de $0, \mathrm{I}_{4}$ au lieu de $0, \mathbf{I} 2$ pour un ajustement par une courbe du second degré.



FIG I

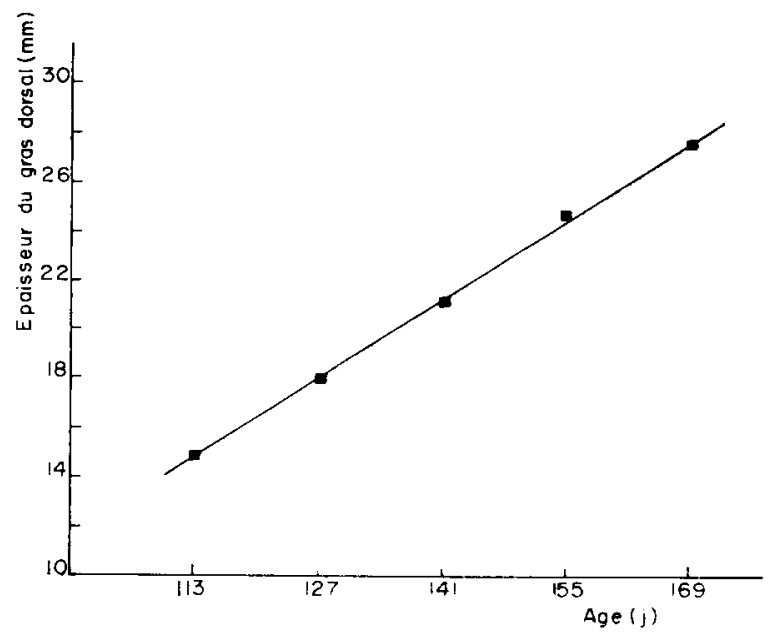

FIG 2

Lìn désignant par X l'âge (en jours), $\mathrm{Y}$ le logarithme décimal du poids (en $\mathrm{kg}$ ) et $Z$ l'épaisseur du gras (en $\mathrm{mm}$ ), les équations des droites de régression sont :

$$
\begin{aligned}
& \mathrm{Y}=0,00572 \mathrm{X}+0,968 \\
& Z=0,23 \mathrm{X}-\mathrm{Ir}, \mathrm{I} 9 \\
& Z=40,86 \mathrm{Y}-5 \mathrm{I}, 24
\end{aligned}
$$


3. Variations individuelles dans la relation entre le poids et l'épaisseur du gras.

La relation (3) décrit l'évolution globale de l'épaisseur du gras en fonction du poids dans l'échantillon étudié. Le problème est de savoir si une relation du même type est applicable aux individus, ce qui rendrait possible de prédire l'épaisseur de gras d'un verrat à Ioo $\mathrm{kg}$ à partir de mesures faites au cours de sa croissance.

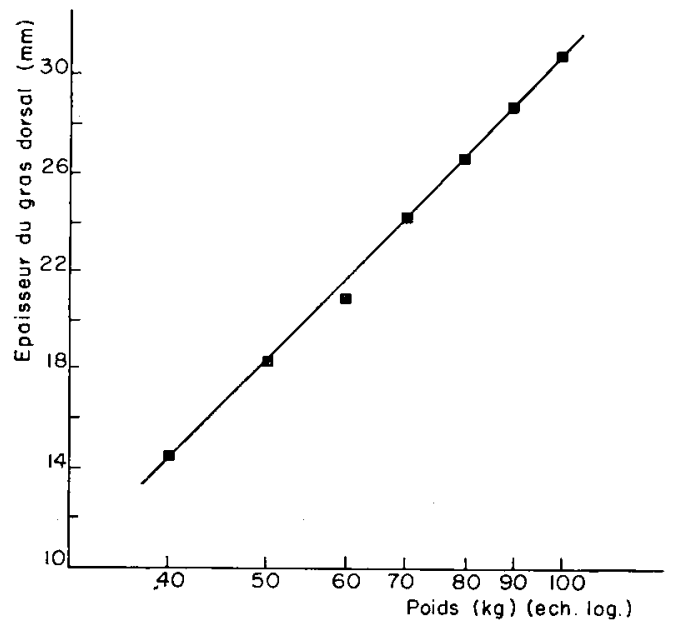

FIG. 3

Le tableau 5 montre que, dès $40 \mathrm{~kg}$ pour le pourcentage de morceaux gras et dès $50 \mathrm{~kg}$ pour le pourcentage de morceaux nobles, des corrélations significatives existent entre l'épaisseur du gras et les résultats de la décotipe des carcasses à Ioo kg.

\section{TABLEAU 5}

Corrélations entre les épaisseurs de gras à différents poids et les résultats de la découpe des carcasses

\begin{tabular}{|c|c|c|c|}
\hline & & $\begin{array}{l}\text { Pourcentage des } \\
\text { morceaux nobles }\end{array}$ & $\begin{array}{l}\text { Pourcentage des } \\
\text { morceaux gras }\end{array}$ \\
\hline 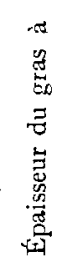 & $\begin{array}{r}40 \mathrm{~kg} \\
50 \mathrm{~kg} \\
60 \mathrm{~kg} \\
70 \mathrm{~kg} \\
80 \mathrm{~kg} \\
90 \mathrm{~kg} \\
100 \mathrm{~kg}\end{array}$ & $\begin{array}{r}-0,32 \\
-0,43 \\
-0,39 \\
-0,59 \\
-0,51 \\
-0,59 \\
-0,62\end{array}$ & $\begin{array}{l}0,43 \\
0,57 \\
0,51 \\
0,60 \\
0,56 \\
0,73 \\
0,82\end{array}$ \\
\hline
\end{tabular}
Valeur du coefficient de corrélation dans
l'hypothése $\rho=0$$\quad\left\{\begin{array}{l}\text { au seuil de } 5 \%: 0,40 \\ \text { au seuil de } 1 \%: 0,52\end{array}\right.$ 
Par ailleurs, nous pouvons tenir compte non seulement de 1'épaisseur du gras à un poids donné mais aussi de l'évolution de cette épaisseur en fonction du poids. Ainsi, en prolongeant, par exemple, la droite passant par les points d'abscisse log 40 et $\log 80$, nous obtenons les résultats suivants :

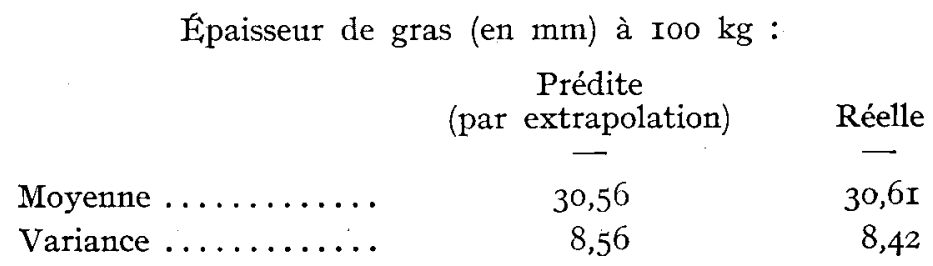

I a corrélation entre l'épaisseur prédite et 1'épaisseur réelle est de 0,86 .

\section{DISCUSSION}

\section{Validité des mesures d'épaisseur de gras sur le vivant et sur la carcasse}

Les auteurs qui ont effectué des mesures aux emplacements proposés par HAzEL. et $\mathrm{KI}_{\mathrm{INE}}$ (I952) ont tous signalé la difficulté des mesures au niveau de l'épaule. En effet, à cet emplacement, qui correspond approximativement à la $5^{\mathrm{e}}$ vertèbre dorsale, se trouve le muscle trapèze qui, d'après BLIN et coll. (I963), s'étend jusqu'au 8 e et ro espace intercostal, et s'enlève généralement avec le lard dans la découpe parisienne. L'écho que renvoie ce muscle est parfois difficile à détecter et'. a lecture correspond alors soit à la face inférieure de ce muscle, soit aux faces supérieures des deux muscles situés immédiatement au-dessous, le long dorsal et le long épineux. L'examen des carcasses de cette expérience et d'autres carcasses soumises aux mêmes mestures a montré que c'était généralement le cas, ce que confirme également l'examen des courbes individuelles d'accroissement qui présentent une allure plus irrégulière à l'épaule qu'au dos et au rein. Les résultats du tableau 4 montrent que, malgré le manque de sûreté des mesures à l'épaule, la moyenne des six mesures. fournit un excellent moyen de prédire la valeur de la carcasse avant l'abattáge. Les valeurs nettement plus faibles des corrélations avec les épaisseurs mesurées sur la carcasse sont sans doute attribuables, pour une large part, au fait que l'échantillon est de taille limitée et de plus tronqué, les verrats les moins gras ayant été retenus pour la reproduction.

\section{Relation entre le poids et l'épaisseur du gras}

La linéarité de cette relation a été admise par HETZER et coll. (I956) entre les poids de 70 et roo kg, par DE PAPE et WHATLEY (I956) entre les âges de 56 et I40 jours par NofrSINGER et coll. (I959) entre les poids de 45 et $90 \mathrm{~kg}$.

La même hypothèse a également été adoptée par S'TANDAL (I962) pour corriger 
en fonction du poids des épaisseurs de gras mesurées sur des pores âgés de Iro à 260 jours. Mais les données qui ont servi à ce dernier auteur ont été recueillies dans de nombreux élevages et ne sont pas directement comparables à des résultats obtenus en milieu uniforme.

Trois causes différentes peuvent expliquer le désaccord de nos résultats avec ceux des auteurs qui viennent d'être cités. Citons d'abord les conditions d'élevage et d'alimentation : il est possible qu'en alimentation ad libitum l'accroissement de l'épaisseur du gras soit plus important et tende à devenir constant en fonction du poids. Le sexe a également une influence certaine, car on sait que la déposition du gras est plus intense chez les mâles castrés et les études précédemment citées (à l'exception de celle de STANDAL) ne portent que sur des mâles castrés et des femelles. Enfin, une dernière cause de désaccord provient des différences dans les intervalles de poids considérés.

\section{Prédiction de la valeur finale de la carcasse i partir de mesures effectuees au cours de l'engraissement}

HE'TZER et coll. (I956) ont été les premiers à signaler l'intérêt de mesures sur le vivant à partir de $80 \mathrm{~kg}$ pour prédire la valeur de la carcasse à roo $\mathrm{kg}$. Ces auteurs concluent même à la supériorité des mesures sur le vivant par rapport aux mesures d'épaisseur de gras effectuées sur la carcasse. De même URBAN et HAzE, (rg6o) estiment que des mesures effectuées environ 5 semaines avant l'abattage permettraient de prédire avec une bonne précision la valeur de la carcasse.

Par contre, DE PAI'E et WhatLEY (I956) considèrent comme inefficaces des mesures prises avant le poids de $90 \mathrm{~kg}$. C'est à une conclusion analogue que parviennent RITTILER et coll. (r964) pour lesquels la connaissance de l'épaisseur du gras dorsal à un stade donné de croissance ne permet pas d'en prédire l'accroissement ultérieur.

D'après l'échantillon limité étudié ici, il semble pourtant qu'une prédiction soit possible assez précocement s'il est tenu compte non seulement de l'épaisseur à un poids donné mais aussi de l'accroissement d'épaisseur observé sur une période déterminée. Ainsi la sélection des verrats sur leur adiposité pourrait s'envisager dès $80 \mathrm{~kg}$, par exemple ; à ce poids, la castration des individus éliminés reste possible sans entraîner de dépréciation de la carcasse si l'abattage intervient à Ioo $\mathrm{kg}$. Il faudrait cependant connaître les héritabilités et corrélations génétiques relatives aux mesures d'épaisseur de gras à différents poids pour évaluer avec précision les gains génétiques qu'il serait possible d'obtenir selon le stade de la croissance auquel s'effectue le choix des reproducteurs.

S'il s'agit de ramener à poids constant des épaisseurs de gras mesurées sur des animaux de poids variable une relation du type de l'équation (3) peut être utilisée, ce qui revient à attribuer à tous les individus le même taux d'accroissement, mais il est préférable, comme cette étude le suggère, de faire au moins deux mesures sur le même individu au cours de sa croissance de manière à estimer les taux d'accroissement individuels. 


\section{SUMMARY}

INCREASE IN THICKNESS OF BACK FAT IN THE GROWING; BOAK

A sample of 29 Large-White boars was individually fed to appetite 3 times daily at the progeny testing station at Jouy-en-Josas. From 40 to $100 \mathrm{~kg}$ liveweight thickness of back fat was measured ultrasonically every 14 days. The relation between thickness of back fat and liveweight differed significantly from linearity but results support the hypothesis that there is a linear relation between thickness of back fat and the logarithm of liveweight. On this basis it is possible to predict thickness of back fat, and hence carcase value at $100 \mathrm{~kg}$ from measurements taken at 40 and $80 \mathrm{~kg}$. The correlation coefficient of thickness of back fat measured at $\mathrm{s}$ oo $\mathrm{kg}$ with values predicted from measureinents at 40 and $80 \mathrm{~kg}$ was $r=0.86$. The same relationship could also be used to adjust to constant weight measurements taken at different liveweights.

\section{RÉFÉRENCES BIBLIOGRAPHIQUES}

Anurews F. N., Whaley R. M., I954. (Cité par Noffsinger at coll., 1959).

Bltn P. C., Labie C., Cle P., Jost P., 1963. Bases anatomiques de la diagnose de provenance régionale des côtelettes de porc. Rec. Med. Vétér., 139, 733-75r.

DE l'APE J. G., Whatley J. $\Lambda ., 1956$. Live hog probes at various sites, weights and ages as indicators of carcass merit. J. animal Sci., 15, 1029-1036.

Dumont B. L., i 957 . Nouvelles méthodes pour l'estimation de la qualité des carcasses sur les porcs vivants Réunion de la F. E. Z. sur l'éprente de descendance des porcs. Copenhague.

Dumont B. L., Février R., 1957. Méthode de mesure radiographique de l'épaisseur des tissus adipeux sous-cutanés chez le porc. Ann. Zootech., 6, 29-40.

FÉvrikr R, 1958. (Données non publiées).

Hazet L. N., KulNe E. A., I952. Mechanical measurement of latness and carcass value on live hogs. J. animal Sci., 11, 313-318

Hetzer H. O., Zeller J. H., Havkins O. G., 1956. Carcass yields as related to live hog probes at various weights and locations. J. animal Sci., 15, 257-270.

MCMEEkan C. P., I940. Growth and development in the pig, with special reference to carcass quality characters. J. agric. Sci., 30, 276-343.

Noffsinger T. L., Andrews F. N., ANuersos V. L., 1959. The rate of fat deposition in four lines of swine. J. animal Sci., 18, 127-133.

Rittler A., Scho e P., Schelder F., Fkwson D., ig6.4. Zur Frage der Genauigkeit von EcholotMessungen am lebenden Schwein in verschiedenen Gewichtabsihnitten. Ziuchtungskunde, 36, I59-168.

Standal N., 1962. A scoring-system for pig selection based on performance testing for backfat thickness and rate of gain. Agric. College Norway. Insitute of Animal Genetics and Breeding. Report $n^{\circ}{ }^{\circ} 8$.

I I RBAN W. E., HAZEL I. N., 1960. Ultrasonic measurement of fatness in swine during the growing-fatteniug period. J. animal Sci., 19. 1228-1229. 